\title{
Changes in White Matter Organization in Adolescent Offspring of Schizophrenia Patients
}

\author{
Max de Leeuw*,', Marc M Bohlken', René CW Mandl', Manon HJ Hillegers', René S Kahn' and Matthijs Vink ${ }^{1,2}$ \\ 'Brain Center Rudolf Magnus, Department of Psychiatry, University Medical Center Utrecht, Utrecht, The Netherlands; 'Departments of \\ Experimental and Developmental Psychology, Faculty of Social Sciences, Utrecht University, Utrecht, The Netherlands
}

\begin{abstract}
Schizophrenia is associated with frontostriatal network impairments underlying clinical and cognitive symptoms. We previously found disruptions in anatomical pathways, including the tract connecting the left nucleus accumbens and left dorsolateral prefrontal cortex (DLPFC). Similar deficits are observed in unaffected siblings of schizophrenia patients, indicating that these deficits are linked to a genetic vulnerability for the disorder. Frontostriatal tract disruptions may arise during adolescence, preceding the clinical manifestation of the disorder. However, to date, no studies have been performed to investigate frontostriatal tract connections in adolescents who are at increased familial risk for schizophrenia. In this study, we investigate the impact of familial risk on frontostriatal tract connections using diffusion tensor imaging in 27 adolescent offspring of schizophrenia patients and 32 matched control adolescents, aged 10-18 years. Mean fractional anisotropy (FA) was calculated for the tracts connecting the striatum (caudate nucleus, putamen, nucleus accumbens) and frontal cortex regions (DLPFC, medial orbital frontal cortex, inferior frontal gyrus). As expected, based on siblings data, we found an impact of familial risk on frontostriatal development: schizophrenia offspring showed increased FA in the tracts connecting nucleus accumbens and DLPFC as compared with control adolescents. Moreover, while FA increased across age in control adolescents, it did not in schizophrenia offspring. We did not find differences in FA in other frontostriatal tracts. These results indicate altered development of white matter in subjects who are at familial risk for schizophrenia and may precede frontostriatal white matter alterations in adult schizophrenia patients and siblings.

Neuropsychopharmacology (2017) 42, 495-50I; doi:I0.I038/npp.2016.130; published online 3 I August 2016
\end{abstract}

\section{INTRODUCTION}

The symptoms and cognitive impairments that are characteristic for schizophrenia may stem from dysfunctions of the frontal lobe and striatum (Howes and Kapur, 2009). Indeed, neuroimaging studies have consistently shown frontostriatal impairments in schizophrenia patients. First, functional MRI studies have demonstrated reduced levels of frontostriatal activation in patients in the context of several cognitive tasks including working memory, reward processing, and inhibition (Morris et al, 2012; van Veelen et al, 2010, 2011; Vink et al, 2006; Zandbelt et al, 2011). Second, patients show decreased frontal and striatal volumes (Emsley et al, 2015; Harms et al, 2010). Finally, diffusion tensor imaging (DTI) studies points in the direction of altered white matter organization in tracts connecting frontal and striatal regions (Bracht et al, 2014; Quan et al, 2013). Importantly, we recently observed such reductions in unaffected siblings of schizophrenia patients (de Leeuw et al, 2015a), suggesting genetic factors underlying frontostriatal dysfunction in

\footnotetext{
*Correspondence: Dr M de Leeuw, Brain Center Rudolf Magnus, University Medical Center Utrecht, Room A01.126, P.O. Box 85500 Utrecht 3508 GA, The Netherlands, Tel: +3I 88 755925I, Fax: +3I 88 7555443, E-mail: m.deleeuw-5@umcutrecht.nl or maxdeleeuw@gmail.com Received I5 February 2016; revised 4 July 2016; accepted I4 July 2016; accepted article preview online 21 July 2016
}

schizophrenia siblings. Specifically, we found decreased fractional anisotropy (FA) in the tract connecting the left nucleus accumbens and left dorsolateral prefrontal cortex (DLPFC) in both patients $(n=24)$ and siblings $(n=30)$ compared with matched controls $(n=58)$, respectively, indicating altered white matter organization (Basser and Pierpaoli, 1996). Consequently, altered frontostriatal white matter organization may represent a genetic vulnerability for schizophrenia. Such an interpretation is in line with other observations of impaired frontostriatal dysfunction in firstdegree relatives of schizophrenia patients (Grimm et al, 2014; de Leeuw et al, 2013, 2015b; Raemaekers et al, 2006; Vink et al, 2006; Zandbelt et al, 2011).

This genetic vulnerability may result in altered frontostriatal development, preceding the overt manifestation of schizophrenia (Paus et al, 2008; Rapoport et al, 2012). Deficits in frontostriatal development may become apparent already during adolescence, when the striatum is fully maturing, and the frontal cortex is developing (Casey et al, 2008; Hoogendam et al, 2013; Vink et al, 2014a, b). Offspring of schizophrenia patients may be best suited to investigate the impact of genetic vulnerability on adolescent brain development, as they inherit on average half their DNA from their ill parent. Indeed, these offspring have a 10 -fold increased incidence of schizophrenia-related psychosis in adulthood (Rasic et al, 2014). They not only share a genetic 


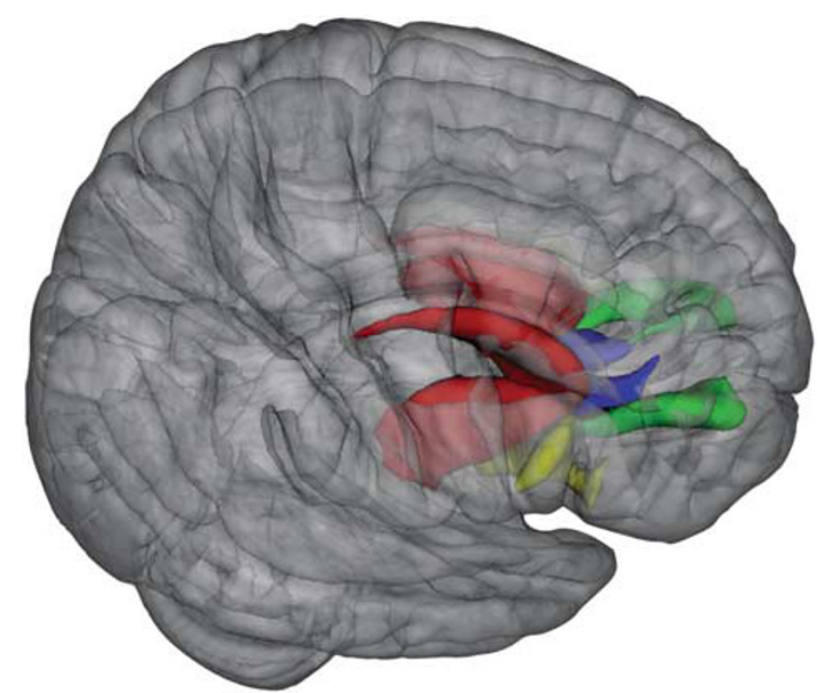

Figure I Mean fractional anisotropy was compared along averaged fibers connecting the striatum (red) with the with frontal cortex regions: striatumdorsolateral prefrontal cortex (green), striatum-medial orbital frontal cortex (blue), and striatum-inferior frontal gyrus (yellow). Right = right.

vulnerability with their parent(s), they are considered to be at increased familial risk as environmental factors also have an important role. Furthermore, and in contrast to clinical atrisk adolescents (Yung et al, 1998), these adolescents are selected based on this familial risk and do not (yet) have a diagnosis of schizophrenia or psychotic symptoms, and do not receive antipsychotic treatment.

We recently reported on diminishing striatal activation across adolescent development during reward anticipation in adolescent offspring of schizophrenia patients using functional MRI (Vink et al, 2016b). This result suggests that genetic vulnerability for schizophrenia (negatively) impacts frontostriatal network function during adolescence. However, to date, no DTI studies have been performed to investigate the impact of this genetic vulnerability on structural frontostriatal tracts in adolescence, although there is one study that reported increased FA values in the striatum in clinical at-risk adolescents who report psychotic experiences (O'Hanlon et al, 2015).

Here, we examined FA in frontostriatal pathways using DTI in 27 adolescent offspring of schizophrenia patients and 32 adolescent offspring of healthy parents. FA measures were investigated in frontostriatal connections, including tracts from striatal subregions (caudate nucleus, putamen, and nucleus accumbens) directed to frontal cortex regions (DLPFC, medial orbital frontal cortex (mOFC) and inferior frontal gyrus (IFG)) and compared between schizophrenia offspring and control adolescents.

We hypothesize altered FA values in frontostriatal tract connections as schizophrenia offspring share genetic vulnerability with their ill parent(s). Specifically, we hypothesize FA changes in the tract connecting the left nucleus accumbens and left DLPFC may be altered during adolescence in schizophrenia offspring, given our previous finding of reduced FA in schizophrenia patients and siblings specifically in this tract (de Leeuw et al, 2015a). Similarly, as we did not observe changes in any other tract in patients and siblings as compared with controls, we hypothesize no FA differences in the other frontostriatal tracts in schizophrenia offspring vs control adolescents.

\section{MATERIALS AND METHODS}

\section{Participants}

Twenty-seven adolescent offspring (14.2 \pm 2.7 years, 8 males $)$ of schizophrenia patients and 32 matched control adolescents $(13.6 \pm 1.8$ years, 17 males) participated in this study. Age did not differ between groups $(t(1,58)=-1.1, p=0.30)$. Although there were less males in the group of adolescent offspring, this difference was not significant $\left(X^{2}=3.3\right.$, $p=0.07)$. All subjects were right-handed. None of the participants received psychotropic medication, had contraindications for MRI, suffered from alcohol or drug dependence (except three schizophrenia offspring were cigarette smokers), had a history of a neurological diagnosis, or psychotic disorder. Current and lifetime DSM-IV axis I disorders were obtained in all participants by a face-to-face interview with both the child and the parent using the Schedule for Affective Disorders and Schizophrenia for School Age Children, Present and Lifetime Version (K-SADS-PL) (Kaufman et al, 2000). DSM-IV axis I disorders and age of onset of parents in the schizophrenia offspring group were ascertained during in-person interviews by the Structured Clinical Interview for DSM-IV Axis I Disorders (First, 1997) and further confirmed by the treating psychiatrist. Control parents were screened for psychiatric disorders by the MINI-SCAN (Nienhuis et al, 2010), before participation of their adolescent offspring, as an affected first-degree relative was an exclusion criterion. Characteristics of participants' socioeconomic status and education are shown in Supplementary Table S1.

Participants received monetary compensation (7.50 euro) for participation. Written informed consent was obtained from both parents or caregivers and offspring older than 12 years of age. The ethics committee of the University Medical Center of Utrecht approved this study.

\section{Diffusion Tensor Imaging}

Image acquisition and preprocessing. A T1-weighted structural MRI scan and a set of two diffusion-weighted scans were obtained from each subject using a $3.0 \mathrm{~T}$ Achieva scanner (Philips, Best, The Netherlands). One three-dimensional T1-weighted scan (185 slices; repetition time $=8.4 \mathrm{~ms}$; echo time $=3.8 \mathrm{~ms}$; flip angle $=8^{\circ}$; field of view, $252 \times 185 \times 288 \mathrm{~mm}^{3}$; voxel size: $1 \mathrm{~mm}$ isotropic) of the whole head was made for anatomical reference. The T1-weighted scans were used to extract anatomically delineated ROI's of the caudate nucleus, putamen, nucleus accumbens, DLPFC (consisting of the rostral middle frontal gyrus; Kikinis et al, 2010), mOFC, and IFG (consisting of the Pars Opercularis, Pars Orbitalis, and Pars Triangularis) (Figure 1) in each hemisphere using the FreeSurfer 5.1.0 structural imaging pipeline (Fischl et al, 2004). See Supplementary Table S2 for the volumetric measurements of the anatomically delineated ROIs.

A set of two transverse diffusion-weighted (DWI) scans were acquired (30 diffusion-weighted volumes with different non-collinear diffusion directions with $b$-factor $=1000 \mathrm{~s} / \mathrm{mm}^{2}$ 


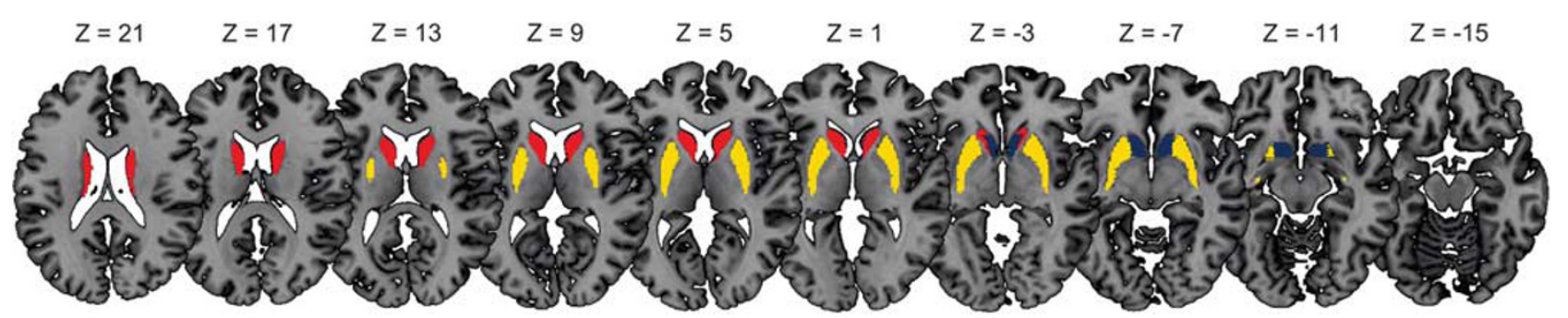

Figure 2 The striatum was divided into three subregions: nucleus accumbens (blue), caudate nucleus (yellow), and putamen (red) and mean fractional anisotropy was computed for tracts originating from these striatal subregions directing to frontal cortex regions including dorsolateral prefrontal cortex, medial orbital frontal cortex, and inferior frontal gyrus. Right $=$ right.

and 8 diffusion-unweighted volumes with $b$-factor $=0 \mathrm{~s} / \mathrm{mm}^{2}$; parallel imaging SENSE factor $=2.5$; flip angle $=90^{\circ}$; 60 slices of $2.5 \mathrm{~mm}$; no slice gap; $96 \times 96$ acquisition matrix; reconstruction matrix $128 \times 128$; FOV $=240 \mathrm{~mm}$; TE $=88 \mathrm{~ms}$; $\mathrm{TR}=9822 \mathrm{~ms}$; no cardiac gating; and total scan duration $=296 \mathrm{~s}$ ). The second DWI scan is identical to the first except that the $k$-space readout is reversed, which allows for correction of susceptibility artifacts during preprocessing. Preprocessing of the DWI scans was performed with the diffusion toolbox of Andersson and Skare (2002) and Andersson et al (2003) and in-house developed software (Mandl et al, 2010). First, susceptibility artifacts were corrected by calculating a distortion map based on the two $b=0$ images acquired with reversed $k$-space readout. Subsequently, it was applied to all DWI volumes. This resulted in one corrected DWI set consisting of a single $b=0$ volume (averaged over $8 b=0$ volumes) and 30 corrected weighted volumes (Andersson et al, 2003). Finally, the DWI set was corrected for eddy-current distortions and small head movements (Andersson and Skare, 2002).

Frontostriatal fiber tractography and diffusion parameter reconstruction. Diffusion modeling and probabilistic tractography were carried out using the FMRIB Diffusion Toolbox (FDT, version 2.0, http://fsl.fmrib.ox.ac.uk/fsl/fsl-4. 1.9/fdt/fdt_probtrackx.html). This process involves generating connectivity distributions from user-specified seed voxels to target voxels. The three anatomical subregions of the striatum (nucleus accumbens, putamen, and caudate nucleus) (Figure 2) were used as seed masks and the three ROI's of the frontal cortex (DLPFC, mOFC, and IFG) were defined as target ROI's (Figure 1). In this way, a total of nine tracts were traced for and within each hemisphere between the frontal cortex and the striatum, leaving 18 fiber distributions for each subject in total. Each frontal ROI was specified as a waypoint and as a termination mask to ensure that only those streamlines running between the seed mask and target ROI were captured in the fiber distribution. The default parameters (5000 streamline samples, step length of $0.5 \mathrm{~mm}$, and curvature threshold of 0.2 ) were used during the probabilistic fiber tracking procedure.

Because the seed points could be volumetrically dependent on individual or group differences, a group average fiber was reconstructed for each of the 18 fiber distributions. First, the Tract Based Spatial Statistics toolbox (version 1.2) (Smith et al, 2006) was applied to subjects' FA maps for warping into FMRIB58_FA standard space. This nonlinear registration was also applied to each of the 18 individually obtained fiber distributions. By only selecting the top $1 \%$ of streamlines in each fiber distribution that overlapped in all participating subjects, a total of 18 group average tracts were reconstructed. The group average tracts were made binary and subsequently they were projected onto the warped FA maps, allowing for the estimation of a mean FA measure per individual per tract. See Supplementary Tables S3 and S4 and Supplementary Figures S1-S3 for additional information on average tract volume and overlap regarding tracts that target the same cortical regions.

Statistical analysis. Multivariate ANOVAs were performed to test for effects of group (schizophrenia offspring and control adolescents) on FA of the tracts connecting the subregions of the striatum (nucleus accumbens, caudatus, and putamen) with the frontal regions (DLPFC, mOFC, and IFG). All results were Bonferroni corrected for multiple testing ( 3 striatal regions $\times 3$ frontal regions $\times 2$ hemispheres $=18$ ), resulting in a critical $p$-value of $0.05 / 18=0.0028$.

\section{RESULTS}

Results are presented in Table 1. FA values for schizophrenia offspring differed from those of adolescent controls only in the tracts connecting the nucleus accumbens and DLPFC (left: $\mathrm{F}(1,58)=10.05, \quad p=0.002$; right: $\mathrm{F}(1,58)=14.09$, $p=0.0004$ ), showing increased mean FA in schizophrenia offspring (FA, left: $0.35 \pm 0.02$; right: $0.36 \pm 0.02$ ) as compared with control adolescents (FA, left: $0.33 \pm 0.02$; right: $0.34 \pm 0.02$ ).

We performed additional analysis to test for potentially confounding effects of gender and movement. First, gender distribution and movement (sum of translation parameters, sum of rotation parameters) did not differ significantly between the groups (gender: $p=0.071$, sum rotations: $p=0.086$, sum translations: $p=0.068$ ). Next, we tested for the effects of gender and movement on FA values. There was a main effect of sum translations on FA for both tracts $(\mathrm{F}(2,52)=3.78, p=0.029)$, indicating that movement has an effect on FA. However, the sum translations by group interaction were not significant, indicating that this effect was equal for the groups. There was no main effect of gender or sum rotations nor a gender or sum rotations by group interaction for these tracts. Finally, our finding of increased mean FA in schizophrenia offspring as compared with control adolescents remained significant when correcting for 
Table I Fractional Anisotropy for Tracts Connecting Subregions of the Striatum with the Frontal Cortex Regions

\begin{tabular}{|c|c|c|c|c|}
\hline & & $\begin{array}{c}\text { Co } \\
(n=32)\end{array}$ & $\begin{array}{c}\text { SZO } \\
(n=27)\end{array}$ & $P$-value \\
\hline \multirow[t]{2}{*}{ Nucleus accumbens-DLPFC } & $\mathrm{R}$ & $0.34 \pm 0.02$ & $0.36 \pm 0.02$ & $0.0004^{\mathrm{a}}$ \\
\hline & $\mathrm{L}$ & $0.33 \pm 0.02$ & $0.35 \pm 0.02$ & $0.002^{\mathrm{a}}$ \\
\hline \multirow[t]{2}{*}{ Nucleus accumbens-mOFC } & $\mathrm{R}$ & $0.21 \pm 0.01$ & $0.21 \pm 0.01$ & 0.19 \\
\hline & $\mathrm{L}$ & $0.19 \pm 0.01$ & $0.19 \pm 0.01$ & 0.19 \\
\hline \multirow[t]{2}{*}{ Nucleus accumbens-IFG } & $\mathrm{R}$ & $0.33 \pm 0.01$ & $0.34 \pm 0.02$ & 0.02 \\
\hline & $\mathrm{L}$ & $0.29 \pm 0.02$ & $0.30 \pm 0.01$ & 0.17 \\
\hline \multirow[t]{2}{*}{ Caudate nucleus-DLPFC } & $\mathrm{R}$ & $0.33 \pm 0.01$ & $0.34 \pm 0.01$ & 0.02 \\
\hline & $\mathrm{L}$ & $0.33 \pm 0.02$ & $0.34 \pm 0.02$ & 0.08 \\
\hline \multirow[t]{2}{*}{ Caudate nucleus-mOFC } & $\mathrm{R}$ & $0.29 \pm 0.01$ & $0.29 \pm 0.01$ & 0.88 \\
\hline & L & $0.27 \pm 0.01$ & $0.27 \pm 0.01$ & 0.48 \\
\hline \multirow[t]{2}{*}{ Caudate nucleus-IFG } & $\mathrm{R}$ & $0.30 \pm 0.02$ & $0.31 \pm 0.01$ & 0.14 \\
\hline & $\mathrm{L}$ & $0.33 \pm 0.02$ & $0.33 \pm 0.01$ & 0.28 \\
\hline \multirow[t]{2}{*}{ Putamen-DLPFC } & $\mathrm{R}$ & $0.36 \pm 0.02$ & $0.37 \pm 0.01$ & 0.004 \\
\hline & $\mathrm{L}$ & $0.37 \pm 0.02$ & $0.38 \pm 0.01$ & 0.04 \\
\hline \multirow[t]{2}{*}{ Putamen-mOFC } & $\mathrm{R}$ & $0.29 \pm 0.01$ & $0.29 \pm 0.01$ & 0.06 \\
\hline & $\mathrm{L}$ & $0.27 \pm 0.02$ & $0.28 \pm 0.01$ & 0.11 \\
\hline \multirow[t]{2}{*}{ Putamen-IFG } & $\mathrm{R}$ & $0.27 \pm 0.01$ & $0.28 \pm 0.01$ & 0.01 \\
\hline & $\mathrm{L}$ & $0.27 \pm 0.02$ & $0.27 \pm 0.01$ & 0.13 \\
\hline
\end{tabular}

Abbreviations: CO, control adolescents; DLPFC, dorsolateral prefrontal cortex; IFG, inferior frontal gyrus; L, left; mOFC, medial orbital frontal cortex; R, right; $\mathrm{SZO}$, schizophrenia offspring.

Values represent mean \pm S.D.

${ }^{\text {a }} P$-values survived Bonferroni correction for multiple testing.

all mentioned covariates. No significant group differences were found in the other tracts projecting from subregions of the striatum to frontal cortex regions.

An explorative analysis on FA values across age for the nucleus accumbens-DLPFC tracts revealed an increase for control adolescents (left: $r=0.6, p=0.001$; right: $r=0.5$, $p=0.01$ ), but not for schizophrenia offspring (left: $r=0.1$, $p=0.6$; right: $r=0.2, p=0.5$ ) (Figure 3 ). This difference between the groups was significant for the left (group by age interaction: $\mathrm{F}(1,55)=6.71, p=0.012)$, but not the right tract $(\mathrm{F}(1,55)=2.49, p=0.12)$.

\section{DISCUSSION}

Here, we show for the first time that FA values in the white matter tracts connecting the nucleus accumbens and the DLPFC are elevated in adolescent offspring of schizophrenia patients (range 10-18 years) when compared with adolescent offspring of healthy parents. Explorative analyses revealed that FA values increased across age in the adolescent controls, but not in the schizophrenia offspring. These results suggest that changes in the formation of this white matter tract are related to the familial risk for schizophrenia. We did not find differences in FA between schizophrenia offspring and controls in other frontostriatal tracts. Taken together, these results are in line with our previous finding of altered white matter organization in adult schizophrenia patients and siblings (de Leeuw et al, 2015a). Longitudinal follow-up of these subjects is needed so that developmental changes of FA measures across age can be coupled with clinical outcomes in adulthood.

To date, no studies have been performed to investigate frontostriatal white matter tracts in adolescent offspring of schizophrenia patients, making it difficult to directly compare our results with the existing literature. However, our finding of increased mean FA in schizophrenia offspring as compared with control adolescents may be in line with one study in adolescent offspring of schizophrenia patients (age range 9-18 years) reporting on increased volumetric measures of subcortical regions across age (Dougherty et al, 2012). Interestingly, a recent population-based case-control study showed that adolescents who reported subclinical psychotic symptoms also had increased FA in striatal regions (O'Hanlon et al, 2015). Although these individuals, in contrast to offspring, are not selected based on a genetic vulnerability, they are clearly at increased risk for developing schizophrenia as they display subclinical psychotic symptoms (Yung et al, 1998).

High FA levels in the nucleus accumbens-DLPFC tract in schizophrenia offspring may indicate suboptimal adaptation to a premorbid process, serving as a marker for either an elevated risk or an effective compensation that is protective against psychosis (Dougherty et al, 2012). The fact that we found an increased FA already at the onset of adolescence points to an altered development before the onset of adolescence (Paus et al, 2008; Rapoport et al, 2012) and may speculatively reflect an advanced peak of myelination (Beaulieu, 2002) or altered development of crossing fibers (Jeurissen et al, 2013) during childhood. This is in line with the idea that genetic factors, in combination with environmental factors, are likely to be at the base of this deficit. For example, aberrations in the gene encoding the dopamine transporter (DAT) gene may contribute to altered FA measures in schizophrenia offspring. Specifically, a study in 41 schizophrenia patients showed an effect of a polymorphism in DAT gene on activation during the verbal fluency task, only in the nucleus accumbens and DLPFC (Prata et al, 2009).

Importantly, in adult-state schizophrenia patients and siblings, we recently found decreased mean FA in the tract connecting the left nucleus accumbens and left DLPFC, representing a vulnerability factor for schizophrenia (de Leeuw et al, 2015a). Interestingly, mean FA in this specific tract in schizophrenia offspring is slightly higher as compared with adult schizophrenia patients and siblings (we used the same analysis and scanner in our paper; de Leeuw et al, 2015a), indicating that FA is likely to decrease after adolescence only in those subjects who will develop schizophrenia or whose sibling will develop the illness. Taken together, genetic vulnerability for schizophrenia may result in frontostriatal neurodevelopmental changes already before adolescence and this may evolve into decreased mean FA during adulthood. 

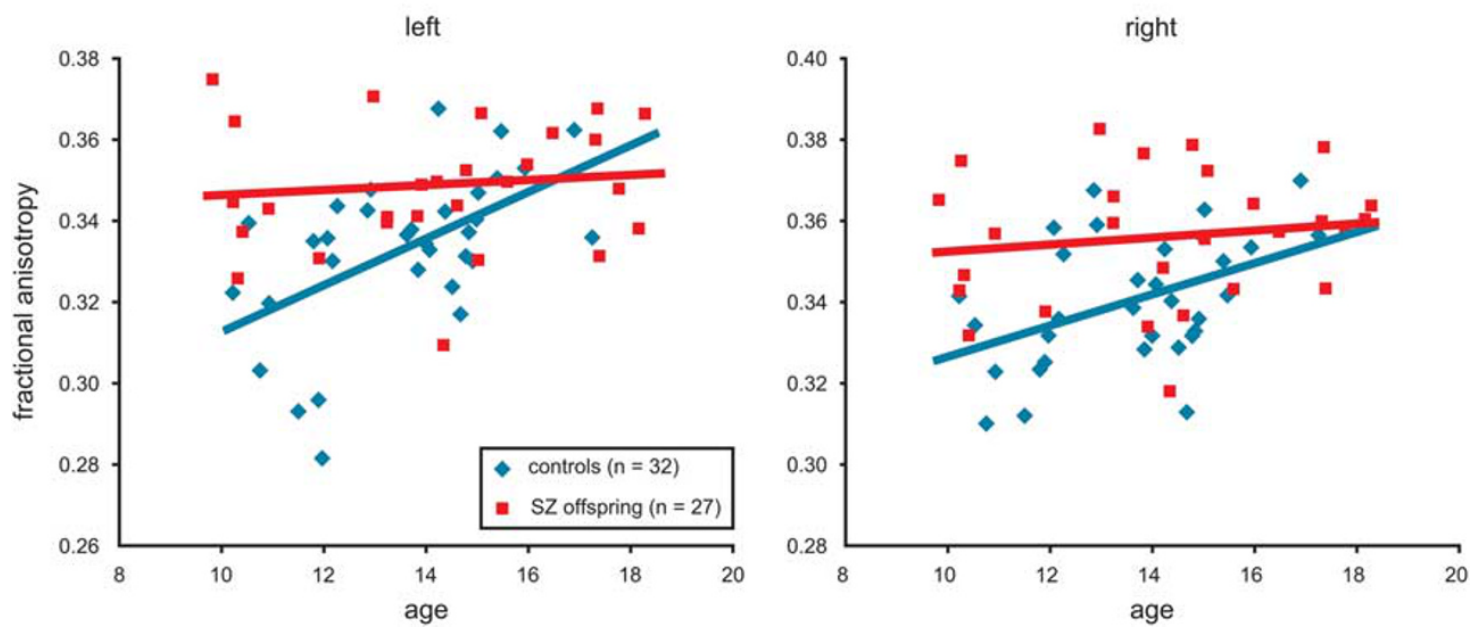

Figure 3 Fractional anisotropy (FA) in the tracts connecting the nucleus accumbens and dorsolateral prefrontal cortex (DLPFC). Mean FA increased across age in control adolescents (in blue) in these tracts, whereas there was no such an increase in offspring of schizophrenia patients (in red). This difference between the groups was significant only for the left tract. Graphs show group trend lines (regression lines), and individual observations plotted against age for offspring of schizophrenia patients (SZ offspring) and control adolescents (controls).

In contrast to decreasing $\mathrm{FA}$ measures in schizophrenia offspring, our finding of an increase in mean FA in the tracts connecting the nucleus accumbens and DLPFC with progressing age in typical developing control adolescents is consistent with previous DTI studies (Giorgio et al, 2008; Lebel et al, 2008; Peper et al, 2013; Peters et al, 2012). As FA may be used as an index for the microstructural directionality of white matter fiber bundles (Basser and Pierpaoli, 1996), our finding of increasing FA across age in control adolescents may reflect maturation of white matter tracts across adolescence. Moreover, control adolescents showed lower mean FA levels as compared with control adults (de Leeuw et al, 2015a), indicating an ongoing increase of FA after adolescence, and this is consistent with a neurodevelopment DTI study in young adults (aged 18-25 years) (Peper et al, 2013). We did not observe such an increase in FA for schizophrenia offspring and this may suggest stagnated white matter fiber maturation during adolescence, possibly as a result of genetic vulnerability for schizophrenia. This finding may be in line with the observation that ventral striatum function did not increase in schizophrenia offspring, whereas it did in control adolescents (Vink et al, 2016b).

The fact that we found in frontostriatal tracts analyses altered FA only in the tract connecting the left nucleus accumbens and left DLPFC matches that of adult schizophrenia patients and siblings (de Leeuw et al, 2015a). This suggests some level of specificity in how the genetic vulnerability for schizophrenia impacts the development of white matter tracts. Indeed, in a discordant twin study genetic overlap between reductions in white matter integrity and schizophrenia liability has been found primarily in frontal and subcortical regions (Bohlken et al, 2016). It may well be that this genetic vulnerability for changes in frontostriatal white matter together with risk alleles impacting dopamine function (Vink et al, 2016a) may contribute to altered FA values in this particular tract. Such a multifaceted origin may also explain why some white matter tracts are not (or less) affected than others. Indeed, none of the other frontostriatal tracts revealed a significant difference between schizophrenia offspring and controls and this is also consistent with results from adult-state schizophrenia patients and siblings (de Leeuw et al, 2015a). Finally, it is important to note that schizophrenia offspring not only share genetic vulnerability but they are at increased familial risk as environmental factors also have an important role.

The fact that the nucleus accumbens-DLPFC tract is affected is anticipated, given that abnormalities in both DLPFC and nucleus accumbens are thought to contribute to underlying clinical symptoms in schizophrenia (Goghari et al, 2010). Moreover, these regions are key components of the reward network (McClure et al, 2004; Sesack and Grace, 2010). We and other have reported on changes in frontostriatal reward processing in schizophrenia patients (Morris et al, 2012; Nielsen et al, 2012), siblings (Grimm et al, 2014; de Leeuw et al, 2015b), and offspring (Vink et al, 2016b).

The reversed polarity acquisition of the diffusion-weighted scans is a robust way of correcting for field distortions. However, as both scans are acquired separately there is a risk that artifacts occur by head motion between acquisitions. Although we did not find movement to impact our betweengroup results, the fact that we did observe a main effect of movement (summed translation parameters) on FA underlines the importance of taking motion parameters into account in diffusion-weighted imaging studies.

Whole-group average tracts were constructed to minimize the possibility that between-group differences in striatal and cortical volumes confounded our main findings. Supplemental volumetric analyses showed no significant differences in striatal and cortical ROI's, except for the right putamen volume, which was significantly decreased in the schizophrenia offspring group. As no between-group differences were observed in the tracts involving the putamen, it is unlikely that this volumetric difference have confounded our main findings.

Our study has several limitations. First, although our cross-sectional study is the first to provide an indication of 
developmental changes in the frontostriatal network during adolescence in schizophrenia offspring, these findings should be replicated in a within-subject longitudinal study to better assess the developmental of white matter tracts. Second, we did not clinically characterize the siblings of schizophrenia offspring. Future studies should explore whether schizophrenia offspring with siblings who have specific clinical characteristics (such as psychosis) have more pronounced white matter changes. Finally, the current study included data of 27 schizophrenia offspring. This is a relative small sample given the age range (10-18 years) that is being examined. The size of the sample also limits potential subgroup analyses.

Here, we show for the first time increased mean FA values in the tracts connecting the nucleus accumbens and DLPFC in adolescent offspring of schizophrenia patients. Moreover, while FA increases across age in control adolescents, it did not in schizophrenia offspring. Such a pattern, although cross-sectional, indicates altered development of white matter in adolescents at familial risk for schizophrenia. No differences in FA were found between schizophrenia offspring and controls in other frontostriatal tracts. Taken together, these results are in line with our previous observation of decreased FA in adult schizophrenia patients and their siblings only in the tract connecting the nucleus accumbens and DLPFC (de Leeuw et al, 2015a). Longitudinal studies are needed to associate developmental trajectories of white matter tracts to clinical outcomes in adulthood. These findings underscore the vulnerability of schizophrenia offspring, who show brain changes even in the absence of clear psychopathology or cognitive impairments.

\section{FUNDING AND DISCLOSURE}

This research was, in part, funded by Brain and Behavior Foundation, an NARSAD independent investigator grant. The authors declare no conflict of interest.

\section{REFERENCES}

Andersson JLR, Skare S (2002). A model-based method for retrospective correction of geometric distortions in diffusionweighted EPI. Neuroimage 16: 177-199.

Andersson JLR, Skare S, Ashburner J (2003). How to correct susceptibility distortions in spin-echo echo-planar images: application to diffusion tensor imaging. Neuroimage 20: 870-888.

Basser PJ, Pierpaoli C (1996). Microstructural and physiological features of tissues elucidated by quantitative-diffusiontensor MRI. J Magn Reson B 111: 209-219.

Beaulieu C (2002). The basis of anisotropic water diffusion in the nervous system-a technical review. NMR Biomed 15: 435-455.

Bohlken MM, Brouwer RM, Mandl RCW, Heuvel MP, Van den, Hedman AM, De Hert M et al (2016). Structural brain connectivity as a genetic marker for schizophrenia. JAMA Psychiatry 73: 11-19.

Bracht T, Horn H, Strik W, Federspiel A, Razavi N, Stegmayer K et al (2014). White matter pathway organization of the reward system is related to positive and negative symptoms in schizophrenia. Schizophr Res 153: 136-142.

Casey BJ, Jones RM, Hare TA (2008). The adolescent brain. Ann NY Acad Sci 1124: 111-126.

Dougherty MK, Gu H, Bizzell J, Ramsey S, Gerig G, Perkins DO et al (2012). Differences in subcortical structures in young adolescents at familial risk for schizophrenia: a preliminary study. Psychiatry Res 204: 68-74.

Emsley R, Asmal L, Plessis S du, Chiliza B, Kidd M, Carr J et al (2015). Dorsal striatal volumes in never-treated patients with first-episode schizophrenia before and during acute treatment. Schizophr Res 169: 89-94.

First M (1997). User's Guide for the Structured Clinical Interview for DSM-IV Axis I Disorders-Clinical Version (CV). American Psychiatric Press: Washington, DC.

Fischl B, Salat DH, Kouwe AJW van der, Makris N, Ségonne F, Quinn BT et al (2004). Sequence-independent segmentation of magnetic resonance images. Neuroimage 23(Suppl 1): S69-S84.

Giorgio A, Watkins KE, Douaud G, James AC, James S, De Stefano N et al (2008). Changes in white matter microstructure during adolescence. Neuroimage 39: 52-61.

Goghari VM, Sponheim SR, MacDonald AW (2010). The functional neuroanatomy of symptom dimensions in schizophrenia: a qualitative and quantitative review of a persistent question. Neurosci Biobehav Rev 34: 468-486.

Grimm O, Heinz A, Walter H, Kirsch P, Erk S, Haddad L et al (2014). Striatal response to reward anticipation: evidence for a systems-level intermediate phenotype for schizophrenia. JAMA Psychiatry 71: 531-539.

Harms MP, Wang L, Campanella C, Aldridge K, Moffitt AJ, Kuelper J et al (2010). Structural abnormalities in gyri of the prefrontal cortex in individuals with schizophrenia and their unaffected siblings. Br J Psychiatry 196: 150-157.

Hoogendam JM, Kahn RS, Hillegers MHJ, Buuren M, van, Vink M (2013). Different developmental trajectories for anticipation and receipt of reward during adolescence. Dev Cogn Neurosci 6: 113-124.

Howes OD, Kapur S (2009). The dopamine hypothesis of schizophrenia: version III-the final common pathway. Schizophr Bull 35: 549-562.

Jeurissen B, Leemans A, Tournier J-D, Jones DK, Sijbers J (2013). Investigating the prevalence of complex fiber configurations in white matter tissue with diffusion magnetic resonance imaging. Hum Brain Mapp 34: 2747-2766.

Kaufman J, Birmaher B, Brent DA, Ryan ND, Rao U (2000). K-SADS-PL. J Am Acad Child Adolesc Psychiatry 39: 1208.

Kikinis Z, Fallon JH, Niznikiewicz M, Nestor P, Davidson C, Bobrow L et al (2010). Gray matter volume reduction in rostral middle frontal gyrus in patients with chronic schizophrenia. Schizophr Res 123: 153-159.

Lebel C, Walker L, Leemans A, Phillips L, Beaulieu C (2008). Microstructural maturation of the human brain from childhood to adulthood. Neuroimage 40: 1044-1055.

de Leeuw M, Bohlken MM, Mandl RCW, Kahn RS, Vink M (2015a). Reduced fronto-striatal white matter integrity in schizophrenia patients and unaffected siblings: a DTI study. NPJ Schizophr 1: 15001.

de Leeuw M, Kahn RS, Vink M (2015b). Fronto-striatal dysfunction during reward processing in unaffected siblings of schizophrenia patients. Schizophr Bull 41: 94-103.

de Leeuw M, Kahn RS, Zandbelt BB, Widschwendter CG, Vink M (2013). Working memory and default mode network abnormalities in unaffected siblings of schizophrenia patients. Schizophr Res 150: 555-562.

Mandl RCW, Schnack HG, Luigjes J, Heuvel MP van den, Cahn W, Kahn RS et al (2010). Tract-based analysis of magnetization transfer ratio and diffusion tensor imaging of the frontal and frontotemporal connections in schizophrenia. Schizophr Bull 36: 778-787.

McClure SM, Laibson DI, Loewenstein G, Cohen JD (2004). Separate neural systems value immediate and delayed monetary rewards. Science 306: 503-507.

Morris RW, Vercammen A, Lenroot R, Moore L, Langton JM, Short B et al (2012). Disambiguating ventral striatum fMRIrelated BOLD signal during reward prediction in schizophrenia. Mol Psychiatry 17: 280-289. 
Nielsen MØ, Rostrup E, Wulff S, Bak N, Lublin H, Kapur S et al (2012). Alterations of the brain reward system in antipsychotic naïve schizophrenia patients. Biol Psychiatry 71: 898-905.

Nienhuis FJ, Van De Willige G, Rijnders CAT, De Jonge P, Wiersma D (2010). Validity of a short clinical interview for psychiatric diagnosis: the mini-SCAN. Br J Psychiatry 196: 64-68.

O'Hanlon E, Leemans A, Kelleher I, Clarke MC, Roddy S, Coughlan H et al (2015). White matter differences among adolescents reporting psychotic experiences: a population-based diffusion magnetic resonance imaging study. JAMA Psychiatry 72: 668-677.

Paus T, Keshavan M, Giedd JN (2008). Why do many psychiatric disorders emerge during adolescence? Nat Rev Neurosci 9: 947-957.

Peper JS, Mandl RCW, Braams BR, De Water E, Heijboer AC, PCMP Koolschijn et al (2013). Delay discounting and frontostriatal fiber tracts: a combined DTI and MTR study on impulsive choices in healthy young adults. Cereb Cortex 23: 1695-1702.

Peters BD, Szeszko PR, Radua J, Ikuta T, Gruner P, Derosse P et al (2012). White matter development in adolescence: diffusion tensor imaging and meta-analytic results. Schizophr Bull 38: $1308-1317$.

Prata DP, Mechelli A, Picchioni MM, Fu CHY, Toulopoulou T, Bramon E et al (2009). Altered effect of dopamine transporter 3' UTR VNTR genotype on prefrontal and striatal function in schizophrenia. Arch Gen Psychiatry 66: 1162-1172.

Quan M, Lee S-H, Kubicki M, Kikinis Z, Rathi Y, Seidman LJ et al (2013). White matter tract abnormalities between rostral middle frontal gyrus, inferior frontal gyrus and striatum in first-episode schizophrenia. Schizophr Res 145: 1-10.

Raemaekers M, Ramsey NF, Vink M, van den Heuvel MP, Kahn RS (2006). Brain activation during antisaccades in unaffected relatives of schizophrenic patients. Biol Psychiatry 59: 530-535.

Rapoport JL, Giedd JN, Gogtay N (2012). Neurodevelopmental model of schizophrenia: update 2012. Mol Psychiatry 2012: 1-11.

Rasic D, Hajek T, Alda M, Uher R (2014). Risk of mental illness in offspring of parents with schizophrenia, bipolar disorder, and major depressive disorder: a meta-analysis of family high-risk studies. Schizophr Bull 40: 28-38.
Sesack SR, Grace AA (2010). Cortico-basal ganglia reward network: microcircuitry. Neuropsychopharmacology 35: 27-47.

Smith SM, Jenkinson M, Johansen-Berg H, Rueckert D, Nichols TE, Mackay CE et al (2006). Tract-based spatial statistics: voxelwise analysis of multi-subject diffusion data. Neuroimage 31: 1487-1505.

Veelen NMJ, van Vink M, Ramsey NF, Buuren M van, Hoogendam JM, Kahn RS (2011). Prefrontal lobe dysfunction predicts treatment response in medication-naive first-episode schizophrenia. Schizophr Res 129: 156-162.

Veelen NMJ, van Vink M, Ramsey NF, Kahn RS (2010). Left dorsolateral prefrontal cortex dysfunction in medication-naive schizophrenia. Schizophr Res 123: 22-29.

Vink M, Derks JM, Hoogendam JM, Hillegers M, Kahn RS (2014a). Functional differences in emotion processing during adolescence and early adulthood. Neuroimage 91: 70-76.

Vink M, de Leeuw M, Luykx JJ, van Eijk KR, van den Munkhof HE, van Buuren $\mathrm{M}$ et al (2016a). DRD2 schizophrenia-risk allele is associated with impaired striatal functioning in unaffected siblings of schizophrenia patients. Schizophr Bull 42: 843-850.

Vink M, de Leeuw M, Pouwels R, van den Munkhof HE, Kahn RS, Hillegers M (2016b). Diminishing striatal activation across adolescent development during reward anticipation in offspring of schizophrenia patients. Schizophr Res 170: 73-79.

Vink M, Ramsey NF, Raemaekers M, Kahn RS (2006). Striatal dysfunction in schizophrenia and unaffected relatives. Biol Psychiatry 60: 32-39.

Vink M, Zandbelt BB, Gladwin T, Hillegers M, Hoogendam JM, Wildenberg WPM van den et al (2014b). Frontostriatal activity and connectivity increase during proactive inhibition across adolescence and early adulthood. Hum Brain Mapp 35: 4415-4427.

Yung AR, Phillips LJ, McGorry PD, McFarlane CA, Francey S, Harrigan S et al (1998). Prediction of psychosis. A step towards indicated prevention of schizophrenia. Br J Psychiatry Suppl 172: 14-20.

Zandbelt BB, Buuren M, van, Kahn RS, Vink M (2011). Reduced proactive inhibition in schizophrenia is related to corticostriatal dysfunction and poor working memory. Biol Psychiatry 70: $1151-1158$.

Supplementary Information accompanies the paper on the Neuropsychopharmacology website (http://www.nature.com/npp) 